\title{
Untangling the atmosphere of the Bullet cluster with Sunyaev-Zel'dovich effect observations
}

\author{
S. Colafrancesco ${ }^{1,2}$, P. Marchegiani ${ }^{3}$, and R. Buonanno ${ }^{4}$
}

\author{
1 INAF - Osservatorio Astronomico di Roma, via Frascati 33, 00040 Monteporzio, Italy \\ e-mail: sergio.colafrancesco@oa-roma.inaf.it \\ 2 on leave from: ASI, V.le Liegi 26, Roma, Italy \\ 3 Dipartimento di Fisica, Università di Roma La Sapienza, P.le A. Moro 2, Roma, Italy \\ e-mail: paolo.marchegiani@oa-roma.inaf.it \\ 4 Dipartimento di Fisica, Università di Roma Tor Vergata, Roma, Italy \\ e-mail: roberto.buonanno@roma2 .infn.it
}

Received 1 November 2010 / Accepted 25 December 2010

\begin{abstract}
Aims. Sunyaev-Zel'dovich effect (SZE) observation of galaxy clusters at high frequency are able to set relevant constraints on intracluster plasma physics because of the strong dependence from the electron distribution function.

Methods. We used the multifrequency SZE observation that are available for the first time up to very high frequencies of $\sim 850 \mathrm{GHz}$ to set contraints on the structure of the Bullet cluster atmosphere. In this context we explore the predictions of five different plasma models with single or multiple temperatures, as well as a model with the coexistence of a thermal background plasma and an additional nonthermal one.

Results. The statistical analysis of the SZE spectrum for the Bullet cluster excludes single temperature models and instead favors a more complex structure of the cluster atmosphere consisting of either two temperature plasma or - more preferably - a thermal plasma at a temperature of $\sim 13.9 \mathrm{keV}$ coexisting with a second plasma component, either at higher temperature or, more preferably, of nonthermal origin, confirming the preliminary, but not conclusive, indications of the hard X-ray observations of the Bullet cluster. Conclusions. The multifrequency study of the SZE signal in the range $\sim 150-850 \mathrm{GHz}$ observed in the Bullet cluster indicates that there is a complex plasma distribution with a combination of a thermal plus a nonthermal electron distribution consistently with the theoretical expectation for a powerful merging in the Bullet cluster.
\end{abstract}

Key words. cosmology: theory - galaxies: clusters: general - galaxies: clusters: individual: Bullet cluster galaxies: clusters: intracluster medium

\section{Introduction}

Comptonization of the CMB photons by electrons in the plasma confined in galaxy clusters (the intracluster plasma) is a powerful probe of the energetics, the energy spectra, and the stratification of their overall electronic distribution. In this context, precise observations of the Sunyaev Zel'dovich effect (hereafter SZE) at $\mathrm{mm}$ wavelengths are crucial for unveiling the detailed structure of cluster atmospheres, their temperature distribution, and the possible presence of suprathermal and/or nonthermal plasma because the high-frequency part (i.e. at $v \gtrsim 350 \mathrm{GHz}$ ) of the SZE spectrum is more sensitive to the relativistic effects of the momentum distribution of the electrons plasma (see, Colafrancesco \& Marchegiani 2007; see also Birkinshaw 1999; Colafrancesco et al. 2003; Colafrancesco 2007, for reviews and references). This is even more so for galaxy clusters with a complex plasma distribution as found, e.g., for powerful merging clusters, like the exemplary case offered by the Bullet cluster (1ES0657-56).

High-frequency SZE observations are becoming available with the advent of microwave and $\mathrm{mm}$ space observatories that can cover the frequency ranges $\sim 600$ to $\sim 1200 \mathrm{GHz}$ with the HERSCHEL-Spire instrument (having also high spatial resolution of 36.6 to $18.1 \mathrm{arcsec}$, respectively) and $\sim 100 \mathrm{GHz}$ to $\sim 850 \mathrm{GHz}$ with the PLANCK-HFI instrument (with spatial resolution of $\sim 4.5$ arcmin).

In particular, the recent HERSCHEL-Spire observation of the SZE at 500, 350, and $250 \mu \mathrm{m}$ (corresponding to $\sim 600,850$, and $1200 \mathrm{GHz}$ ) in the direction of the Bullet cluster (Zemcov et al. 2010) opens the way to a much deeper astrophysical exploration of the atmosphere of this merging cluster by means of spatially resolved spectral studies in frequency regions where the relativistic effects and the sensitivity to the physical parameters of the electron distribution function is enhanced. Lower frequency measurements of the SZE towards the Bullet cluster are also available and are very useful for constraining the total Comptonization factor.

An overall SZE effect signal has been detected towards the Bullet cluster with ACBAR at 150 and $275 \mathrm{GHz}$ (see Gomez et al. 2004), even though the cluster is an unresolved source for this instrument (the ACBAR beam is $\sim 4.5$ arcmin), confirming the early detection of the SZE signal obtained with the SEST telescope at $150 \mathrm{GHz}$ (Andreani et al. 1999). At $150 \mathrm{GHz}$ a CMB brightness decrement has been also found by APEX (Halverson et al. 2009) and by the SPT experiment (Plagge et al. 2010) with a central value of the SZE consistent with the previous findings.

At radio frequencies, the SZE brightness is expected to be quite low and observations obtained with ATCA at $18 \mathrm{GHz}$ also 
show evidence of substructure in the map of the SZE towards the Bullet cluster (Malu et al. 2010), indicating that the intracluster plasma of the Bullet cluster appears to have a significant pressure distribution structure with local peaks that are distributed not fully coincident with the peaks of the plasma emission measure, the galaxy, and the dark matter distribution.

The enriched view of the multifrequency spectrum of the CMB photon Comptonization in the atmosphere of the Bullet cluster today allows a first analysis of the complex plasma structure of this merging cluster in order to disentangle the physical structure of its atmosphere.

We discuss in this paper the constraints that the multifrequency SZE data of the Bullet cluster can set on the different plasma models for its leptonic atmosphere. In Sect. 2 we discuss first the spectral analysis of the SZE signals for five different plasma models and then the spatial analysis that is mainly based on HERSCHEL-Spire data. We discuss in Sect. 3 the statistical analysis of the SZE spectral modeling in comparison with X-ray constraints and then draw our conclusions.

Throughout the paper, we use a flat, vacuum-dominated cosmological model with $\Omega_{\mathrm{m}}=0.3, \Omega_{\Lambda}=0.7$, and $H_{0}=$ $70 \mathrm{~km} \mathrm{~s}^{-1} \mathrm{Mpc}^{-1}$.

\section{The SZE in the Bullet cluster}

The spectral distortion of the CMB spectrum observable in the direction of the galaxy cluster is

$$
\Delta I(x)=2 \frac{\left(k_{\mathrm{B}} T_{\mathrm{CMB}}\right)^{3}}{(h c)^{2}} y \tilde{g}(x),
$$

where $\Delta I(x)=I(x)-I_{0}(x), I(x)$ is the up-scattered CMB spectrum in the direction of the cluster and $I_{0}(x)$ is the unscattered $\mathrm{CMB}$ spectrum in the direction of a sky area contiguous to the cluster. Here $x \equiv h v / k_{\mathrm{B}} T_{\mathrm{CMB}}$, and $T_{\mathrm{CMB}}=2.726 \mathrm{~K}$ is the $\mathrm{CMB}$ temperature today. The Comptonization parameter $y$ is

$$
y=\frac{\sigma_{\mathrm{T}}}{m_{\mathrm{e}} c^{2}} \int P_{\mathrm{e}} \mathrm{d} \ell,
$$

in terms of the pressure $P_{\mathrm{e}}$ contributed by the electronic population. Here $\sigma_{\mathrm{T}}$ is the Thomson cross section, $m_{\mathrm{e}}$ the electron mass, and $c$ the speed of light. The spectral function $\tilde{g}(x)$ of the SZE writes as

$$
\tilde{g}(x)=\frac{m_{\mathrm{e}} c^{2}}{\left\langle\varepsilon_{\mathrm{e}}\right\rangle}\left\{\frac{1}{\tau_{\mathrm{e}}}\left[\int_{-\infty}^{+\infty} i_{0}\left(x e^{-s}\right) P(s) d s-i_{0}(x)\right]\right\}
$$

in terms of the photon redistribution function $P(s)$ and of

$$
i_{0}(x)=I_{0}(x) /\left[2\left(k_{\mathrm{B}} T_{\mathrm{CMB}}\right)^{3} /(h c)^{2}\right]=x^{3} /\left(e^{x}-1\right) .
$$

The quantity

$$
\left\langle\varepsilon_{\mathrm{e}}\right\rangle \equiv \frac{\sigma_{\mathrm{T}}}{\tau_{\mathrm{e}}} \int P_{\mathrm{e}} \mathrm{d} \ell=\int_{0}^{\infty} \mathrm{d} p f_{\mathrm{e}}(p) \frac{1}{3} p v(p) m_{\mathrm{e}} c,
$$

where $f_{\mathrm{e}}(p)$ is the normalized electron momentum distribution function, is the average energy of the electron plasma (see Colafrancesco et al. 2003). The optical depth of the electron population along the line of sight $\ell$ is

$$
\tau_{\mathrm{e}}=\sigma_{\mathrm{T}} \int \mathrm{d} \ell n_{\mathrm{e}} .
$$

The photon redistribution function $P(s)$, with $s=\ln \left(v^{\prime} / v\right)$ in terms of the CMB photon frequency increase factor $v^{\prime} / v$, can be calculated by repeated convolution of the single-scattering redistribution function, $P_{1}(s)=\int \mathrm{d} p f_{\mathrm{e}}(p) P_{\mathrm{s}}(s ; p)$, where $P_{s}(s ; p)$ depends on the physics of inverse Compton scattering. The previous description is general enough to be applied to both thermal and nonthermal plasma, as well as to a combination of the two (see Colafrancesco et al. 2003, for details).

\subsection{Spectral analysis}

We start noticing that a single temperature plasma with $k T=$ $13.9 \mathrm{keV}$ and $\tau=1.3 \times 10^{-2}$, as used by Zemcov et al. (2010) to describe the SZE spectrum at the center of the Bullet cluster, does not fit the observed spectrum well, especially the highfrequency data at $350 \mu \mathrm{m}$ (see Fig. 1). In fact, the $\chi^{2}$ value in this case is 5.30 with 3 degrees of freedom (d.o.f.) (see Table 1).

There are various possibilities for improving the fit of the SZE spectrum: i) inclusion of multiple scatterings; ii) a higher temperature of the plasma; iii) a combination of two thermal plasmas; iv) a combination of a thermal plasma and a nonthermal plasma.

We verified that, for a plasma with $k T=13.9 \mathrm{keV}$ and $\tau=1.3 \times 10^{-2}$, the second-order corrections in $\tau$ to the spectral shape (see Colafrancesco et al. 2003, for technical details) do not produce appreciable variations with respect to the firstorder calculation of the SZE spectrum. This means that the leptonic atmosphere of the Bullet cluster is still in the optically thin regime, for which $\mathrm{CMB}$ photons do not suffer, on average, more than one scattering off the electron population. Since including multiple scattering is not important for calculating SZE in the Bullet cluster, we work in the rest of the paper with the first order approximation in the optical depth. In such a case, the SZE depends separately on the electron optical depth and on the shape of the electrons spectrum: this fact allows us to consider the optical depth and the temperature (or the parameters of the power law for the nonthermal SZE) as independent parameters, thereby simplifying the fitting procedure. Moreover, in the presence of two populations, the overall SZE is simply given by the sum of the two separate effects (see Colafrancesco et al. 2003, for details).

Figure 1 shows the thermal SZE spectrum produced at the center of the cluster's atmosphere, as identified by the HERSCHEL-Spire observation, with a higher plasma temperature $k T=22 \mathrm{keV}$. In this case, the spectrum is marginally consistent with the multifrequency data for a value of the optical depth $\tau=8.3 \times 10^{-3}$, and the $\chi^{2}$ value in this case is 1.59 with 2 d.o.f. (see Table 1). However, this high central temperature does not seem consistent with a single X-ray spectroscopic temperature of $13.9 \pm 0.7 \mathrm{keV}$ as derived by Chandra (see, e.g., Govoni et al. 2004).

The X-ray temperature map of the Bullet cluster shows evidence of a wide range of temperatures, from 8 to more than $22 \mathrm{keV}$ (see discussion by Gomez et al. 2004), as is expected in powerful mergers, and therefore we explore the possibility to improve the SZE spectral fit by a combination of multiple temperature plasma. Figure 2 shows the case for a combination of two thermal plasma with temperatures of 9 and $25 \mathrm{keV}$. The total SZE spectrum fits the data with a $\chi^{2}$ of 1.25 with 0 d.o.f. (see Table 1). Even though the formal fit offered by this solution is compatible with the data, the available number of independent data points (four) is equal to the number of parameters, and the statistical significance of the fit is rather low.

To decrease the number of free parameters, we fix the first plasma temperature at the value of the spectroscopic one derived 


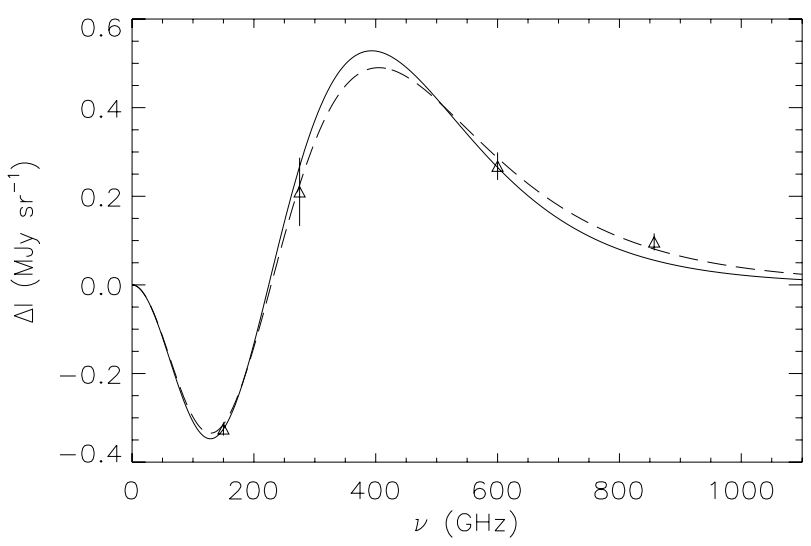

Fig. 1. The SZE spectrum of the Bullet cluster fitted with a singletemperature plasma with $k T=13.9 \mathrm{keV}$ and $\tau=1.3 \times 10^{-2}$, as in Zemcov et al. (2010) (solid), and with a higher temperature plasma with $k T=22 \mathrm{keV}$ and $\tau=8.3 \times 10^{-3}$ (dashed).

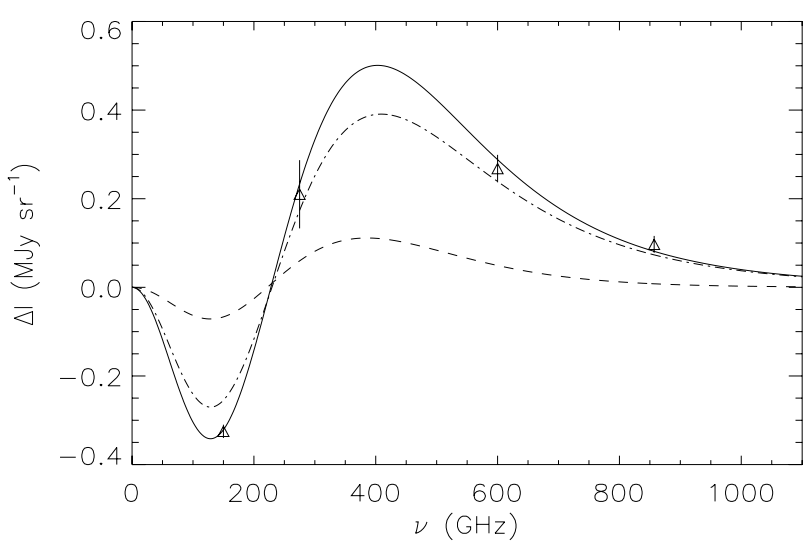

Fig. 2. The SZE spectrum at the center of the Bullet cluster produced by the combination of two thermal plasmas: thermal plasma with $k T=$ $9 \mathrm{keV}$ and $\tau=4 \times 10^{-3}$ (dashed); thermal plasma with $k T=25 \mathrm{keV}$ and $\tau=6 \times 10^{-3}$ (dot-dashed); total SZE induced by the sum of the two thermal plasmas (solid).

by X-ray observations, i.e. $k T=13.9 \mathrm{keV}$, leaving only its optical depth free. Figure 3 shows the case of a combination of two thermal plasma with temperatures of $13.9 \mathrm{keV}$ and $25 \mathrm{keV}$. The total SZE spectrum fits the data with a $\chi^{2}$ of 1.27 with 1 d.o.f. (see Table 1), showing that it is possible to find a combination of optical depths and temperatures that is able to fit the whole data set.

Since powerful merging shocks in clusters can also produce acceleration of particles up to relativistic (or subrelativistic) energies (see, e.g., Dogiel et al. 2007; Wolfe \& Melia 2006, 2008), we also explore the possibility to fit the SZE spectrum with a combination of a thermal background plasma and a nonthermal electron distribution. Figure 4 (upper panel) shows the case of the combination of a thermal plasma with $k T=13.9 \mathrm{keV}$ and $\tau=1.1 \times 10^{-2}$ with an additional nonthermal relativistic plasma with a power-law electron spectrum $N(p) \propto p^{-s}$, with a minimum momentum $p_{1}=1$, a spectral index $s=2.7$ (these two parameters are fixed a priori), and $\tau=2.3 \times 10^{-4}$. This

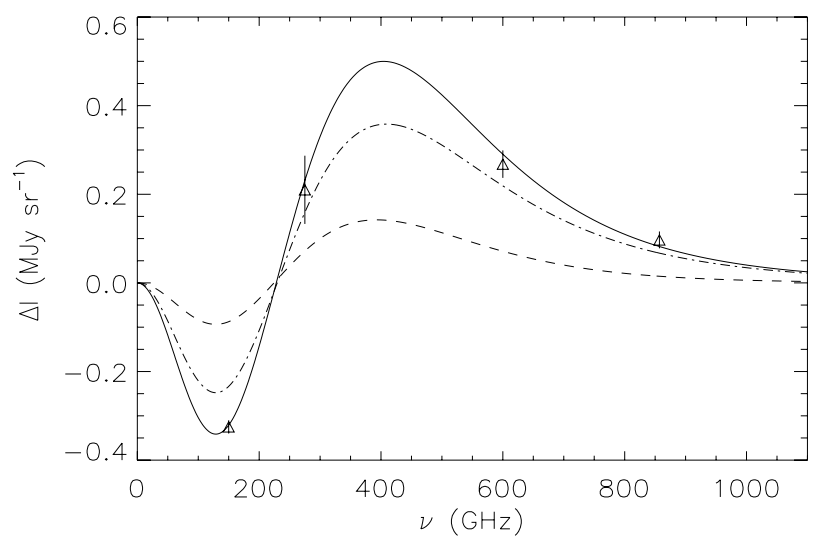

Fig. 3. Same as Fig. 2 but with different parameters: thermal plasma with $k T=13.9 \mathrm{keV}$ and $\tau=3.5 \times 10^{-3}$ (dashed); thermal plasma with $k T=25 \mathrm{keV}$ and $\tau=5.5 \times 10^{-3}$ (dot-dashed); total SZE induced by the sum of the two thermal plasmas (solid).

combination is the one that fits the data best with a $\chi^{2}$ of 0.44 with 2 d.o.f. (see Table 1).

We also consider the case in which the acceleration processes generates a suprathermal tail in the electron distribution (see, e.g., Dogiel et al. 2007). To analyze this last case, we considered a thermal electron population with $k T=13.9 \mathrm{keV}$ for $p \leq p^{*}$ and a nonthermal tail for $p>p^{*}$, and we used the value of $p^{*}$ as a free parameter in the fit to the SZE data. We used two different parametrizations for the supra-thermal tail: i) a power-law momentum distribution, $f_{\mathrm{e}}(p) \propto p^{-s}$, where we fix the value of $s=1.86$ to fit the slope of the hard X-ray tail observed by Swift-BAT (Ajello et al. 2010) with a non-thermal bremsstrahlung emission model; ii) a spectral shape of the momentum distribution for $p>p^{*}$ derived from the solution of the insitu acceleration process of subrelativistic electrons in galaxy clusters (see eq.18 in Dogiel et al. 2007). The best fit to the data obtained with these two models (see Fig. 4, lower panel) are $\chi^{2}=0.75$ and 0.81 with 2 d.o.f. in both cases. It is therefore rather difficult to distinguish these two cases on the basis of the available SZE data. We stress, however, that an additional observation of the Bullet cluster at $v \sim 400 \mathrm{GHz}$ with a precision $\lesssim 1 \%$ of the expected signal (i.e. with a precision $\lesssim 0.01 \mathrm{MJy} / \mathrm{sr}$ or better) will be able - together with the existing data, especially the high-frequency ones - to provide further and, likely crucial, constraints to the suprathermal and/or nonthermal electron population in the Bullet cluster. The combined use of SZE and X-ray measurements can provide further information on this question.

Even though we are well aware of the pitfalls due to the statistics of small data sets, we can exclude according to the $\chi^{2}$ analysis that a single temperature plasma (the two Models 1 and 2 in Table 1) is able to explain the SZE spectrum of the Bullet cluster. As for the double-component models (i.e. Models 3,4 , and 5 in Table 1) the formal statistical analysis does not allow determining the best-fitting model. However, the rms figure of merit of Model 5, which is a factor $\sim 2$ smaller than that of Models 3 and 4, suggests that a combination of a thermal plus a nonthermal plasma provides the best description of the multifrequency SZE data and should, therefore, be considered in more detail as a realistic description of the structure of the atmosphere of the Bullet cluster. 
Table 1. The values of $\chi^{2}$ for the various models used to fit the SZE spectrum of the Bullet cluster.

\begin{tabular}{lcccccccc}
\hline \hline Model & Plasma structure & $k T_{1}$ & $k T_{2}$ & $\tau_{1}$ & $\tau_{2} *$ & $s, p_{1}$ & $\chi^{2}$ & d.o.f. \\
\hline Model 1 & thermal: single T & 13.9 & - & $1.1 \times 10^{-2}$ & - & - & 5.30 & 3 \\
Model 2 & thermal: single T & 22.0 & - & $8.3 \times 10^{-3}$ & - & - & 1.59 & 2 \\
Model 3 & thermal: double T & 9 & 25.0 & $4.0 \times 10^{-3}$ & $6.0 \times 10^{-3}$ & - & 1.25 & 0 \\
Model 4 & thermal: double T & 13.9 & 25.0 & $3.5 \times 10^{-3}$ & $5.5 \times 10^{-3}$ & - & 1.27 & 1 \\
Model 5 & thermal + non-thermal & 13.9 & - & $1.1 \times 10^{-2}$ & $2.3 \times 10^{-4}$ & $2.7,1$ & 0.44 & 2 \\
\hline
\end{tabular}

Notes. The optical depth of the second plasma component (thermal or nonthermal, depending on the model).
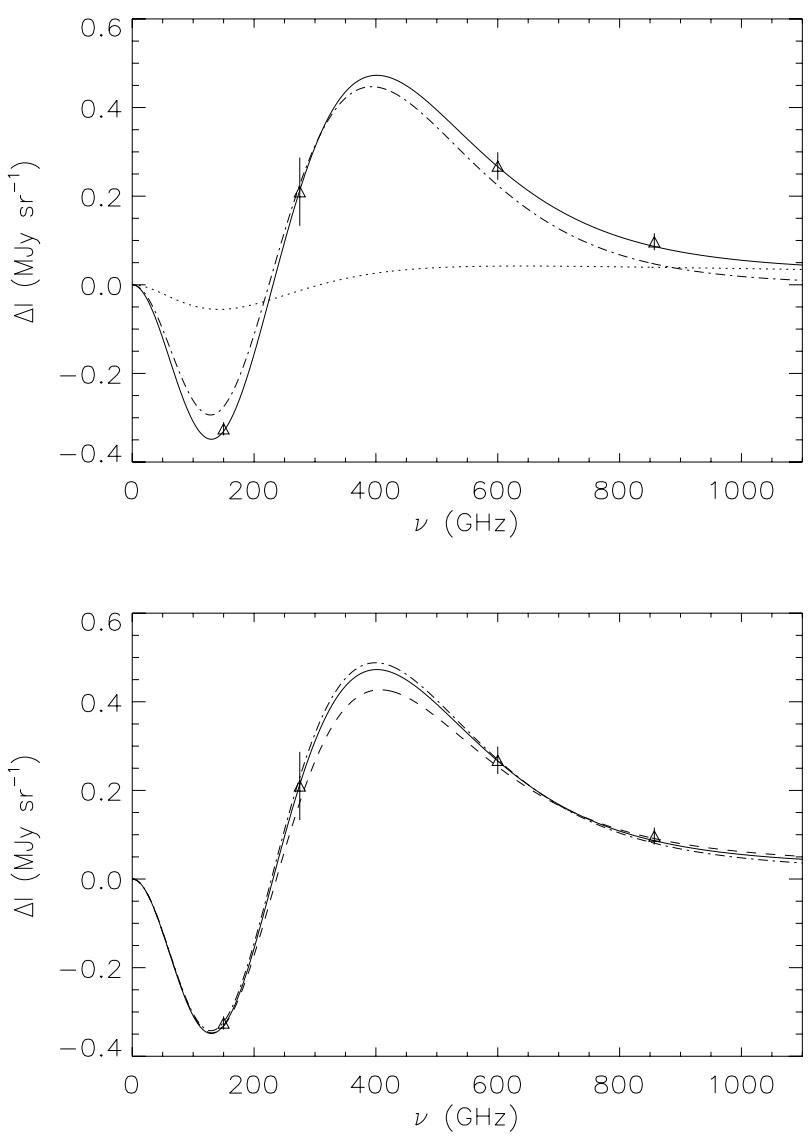

Fig. 4. Upper panel: the SZE spectrum at the Bullet cluster center modeled with a thermal plus nonthermal plasma: thermal plasma with $k T=13.9 \mathrm{keV}$ and $\tau=1.1 \times 10^{-2}$ (dot-dashed); nonthermal plasma with $p_{1}=1, s=2.7$ and $\tau=2.3 \times 10^{-4}$ (dotted); total SZE produced by the sum of the two plasmas (solid). Lower panel: the total SZE produced by the sum of thermal and nonthermal plasma as in the upper panel (solid line); thermal plus a power-law tail with $p^{*}=0.65$, $s=1.86$ and $\tau=1 \times 10^{-2}$ (dashed line); thermal plus tail as in Eq. (18) in Dogiel et al. (2007) with $p^{*}=0.7, \alpha=1 \times 10^{-3}$ (fixed parameter), and $\tau=1.15 \times 10^{-3}$ (dot-dashed line).

\subsection{Spatial analysis}

Figure 5 shows the comparison of the HERSCHEL-Spire brightness profile of the Bullet cluster (Zemcov et al. 2010) (renormalized as to match our zero level to the data zero level) with the predictions of two models:

i) a radial brightness profile produced by a thermal plasma with the parameters derived by Halverson et al. (2009), with an arbitrary normalization;
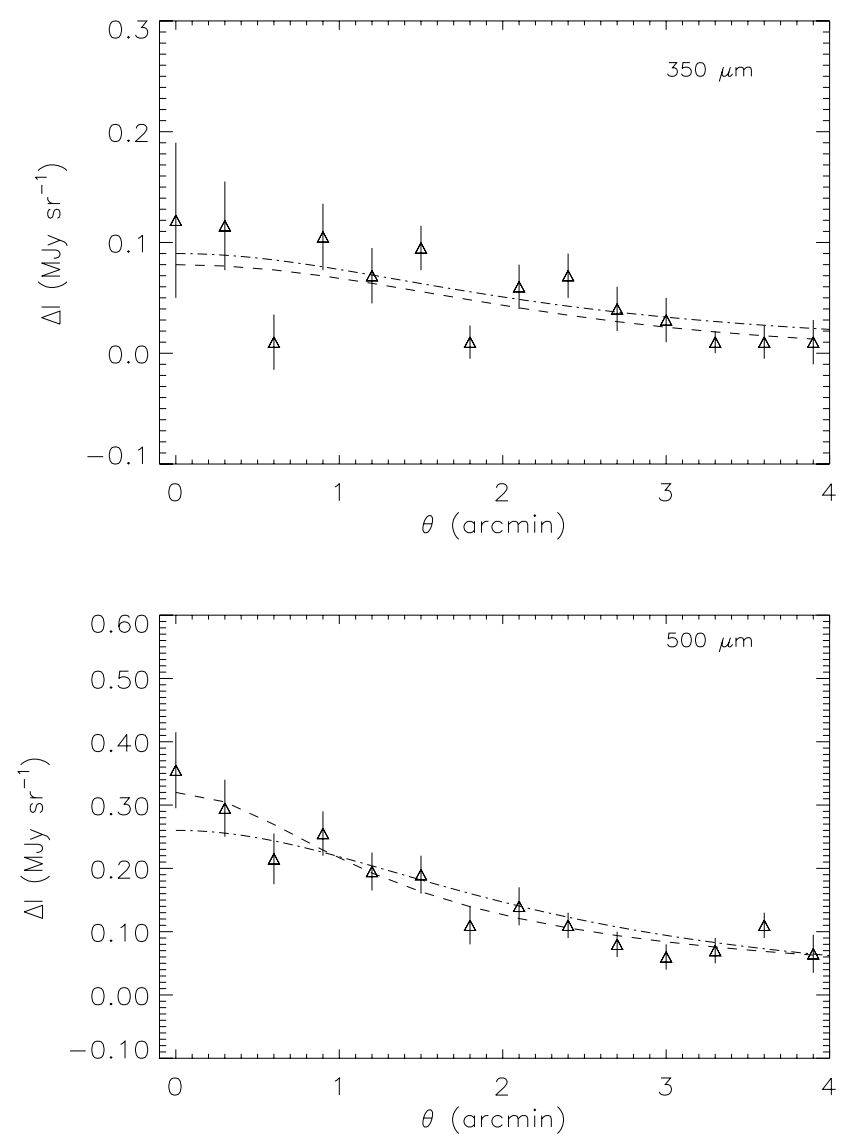

Fig. 5. The SZE brightness radial profile of the Bullet cluster at $350 \mu \mathrm{m}$ (upper panel) and at $500 \mu \mathrm{m}$ (lower panel). The dot-dashed curve corresponds to the form of the brightness profile produced by a thermal plasma adopting the structure parameters given by Halverson et al. (2009), with arbitrary normalization. The dashed curve corresponds to the fit to the data obtained using a model as in Eq. (7), with $S_{0}=$ $0.08 \pm 0.02 \mathrm{MJy} \mathrm{sr}^{-1}, \theta_{\mathrm{c}}=3.96 \pm 1.84 \mathrm{arcmin}$, and $q^{\prime}=2.7 \pm 1.8$ for $350 \mu \mathrm{m}$, and $S_{0}=0.32 \pm 0.04 \mathrm{MJy} \mathrm{sr}^{-1}, \theta_{\mathrm{c}}=1.05 \pm 0.12 \mathrm{arcmin}$, and $q^{\prime}=0.6 \pm 0.3$ for $500 \mu \mathrm{m}$.

ii) a fit to the data using a spatial model as in the following

$$
S(\theta)=S_{0}\left(1+\frac{\theta^{2}}{\theta_{\mathrm{c}}^{2}}\right)^{-q^{\prime}} .
$$

The fit at $350 \mu \mathrm{m}$ has a $\chi^{2}=25.8$ (with 11 d.o.f.), while the one at $500 \mu \mathrm{m}$ has a $\chi^{2}=11.2$ (with 11 d.o.f.). We notice that the two data points at 0.6 and 1.8 arcmin (which are much lower than the extrapolation done from the rest of the data set) make the first fit much worse than the second one. 
We also notice that, in the first case, the core radius is about the maximum dimension of the region within which the HERSCHEL-Spire data have been obtained, and therefore the value of the parameter $q^{\prime}$, which sets the slope of the brightness profile at a large distance from the plasma geometrical center, is not highly significative.

We conclude that the spatial analysis of the SZE signal done at the two frequency at which HERSCHEL-Spire detected the SZE signal (i.e. 600 and $850 \mathrm{GHz}$ ) is not able to set strong constraints on the plasma model for the Bullet cluster. Both spatial profiles are compatible with a unique $\beta$-like radial profile similar to the one produced by the thermal gas alone, even though the two profiles are rather irregular in shape.

\section{Discussion and conclusions}

Powerful merging events in galaxy clusters are likely to produce a complex structure of the cluster atmosphere. Various studies of the temperature structure of the intracluster plasma in the Bullet cluster point, in fact, to a complex temperature distribution of the plasma, with values ranging from 8 to more than $22 \mathrm{keV}$ (Gomez et al. 2003; Zhang et al. 2006) and a central spectroscopic temperature of $k T_{\text {spec }}=13.9 \pm 0.7 \mathrm{keV}$ within $0.5 \mathrm{Mpc}$ of the cluster center (Govoni et al. 2004).

Hard X-ray observations of this cluster with RXTE and XMM-Newton (Petrosian et al. 2006) and with the Swift-BAT (Ajello et al. 2010) also indicate there is a second plasma component in addition to a background thermal one. The previous authors find that the fit to the X-ray data improves over the single temperature plasma case by assuming either the presence of two thermal populations or the combination of a thermal and a nonthermal population, with a statistical preference for the second case.

The merging suffered by the Bullet cluster could have indeed produced an additional high- $T$ plasma distribution if the electron acceleration time scale at the merging shocks were longer than their equilibration time scale (the condition found by the results of Wolfe \& Melia 2006), or if an additional nonthermal population produced either in a merging process with a very short acceleration time scale or by secondary electrons produced by p-p collisions after the high- $E$ protons have been accelerated by the merging and accumulate in the cluster region on long time scales (see, e.g., Wolfe \& Melia 2008). The quasi-stationary case provided by the competition between particle thermalization and their stochastic acceleration and momentum diffusion (Dogiel et al. 2007) can develop a subrelativistic electron distribution tail and can produce suprathermal (or nearly nonthermal) regions in the cluster atmosphere.

Our study of the SZE signal observed in the Bullet cluster arrives at the same conclusion for the atmosphere structure of this cluster: the analysis of the multifrequency SZE spectrum towards the cluster indeed indicates the presence of a thermal plasma at $\sim 13.9 \mathrm{keV}$ coexisting with a second plasma component, either at higher temperature or, more preferably, of a nonthermal origin.

Further and more precise spatially resolved spectroscopic observations of galaxy clusters in the frequency range from $\sim 100 \mathrm{GHz}$ to $\sim 1 \mathrm{THz}$ (available in the near future) will greatly improve our understanding of the structure of the cluster atmospheres through analysis of the SZE signal.

\section{References}

Ajello, M., Rebusco, P., Cappelluti, N., et al. 2010, ApJ, 725, 1688 Andreani, P. Böhringer, H., dall'Oglio, G., et al. 1999, ApJ, 513, 23 Birkinshaw, M. 1999, Phys. Rep., 310, 97

Colafrancesco, S. 2007, New Astron. Rev., 51, 394

Colafrancesco, S., \& Blasi, P. 1998, Astropart. Phys., 9, 227

Colafrancesco, S., \& Marchegiani, P., 2010, A\&A, 520, A31

Colafrancesco, S., Marchegiani, P., \& Palladino, E. 2003, A\&A, 397, 27

Dogiel, V., Colafrancesco, S., Ko, C. M., et al. 2007, A\&A, 461, 433

Gomez, P., Romer, A. K., Peterson, J. B., et al. 2004, AIPC, 703, 361

Govoni, F., Markevitch, M., Vikhlinin, A., et al. 2004, ApJ, 605, 695

Halverson, N. W., Lanting, T., Ade, P. A. R., et al. 2009, ApJ, 701, 42

Malu, S. S., Subrahmanyan, R., Wieringa M., \& Narasimha, D. 2010 [arXiv: 1005.1394$]$

Petrosian, V., Madejski, G., \& Luli, K., 2006, ApJ, 652, 948

Plagge, T., Benson, B. A., Ade, P. A. R., et al. 2010, ApJ, 716, 1118

Wolfe, B., \& Melia, F. 2006, ApJ, 638, 125

Wolfe, B., \& Melia, F. 2008, ApJ, 687, 193

Zemcov, M., Rex, M., Rawle, T. D., et al. 2010, A\&A, 518, L16

Zhang, Y.-Y., Böhringer, H., Finoguenov, A., et al. 2006, A\&A, 456, 55 